\title{
Valley-symmetry-preserved transport in ballistic graphene with gate-defined carrier guiding
}

\author{
Minsoo Kim ${ }^{1}$, Ji-Hae Choi ${ }^{1}$, Sang-Hoon Lee ${ }^{1}, K$ Kenji Watanabe ${ }^{2}$, Takashi Taniguchi ${ }^{2}$, Seung-Hoon Jhi ${ }^{1}$ \\ and Hu-Jong Lee ${ }^{1 \star}$
}

Ever since the discovery of graphene', valley symmetry and its control ${ }^{2,3}$ in the material have been a focus of continued studies in relation to valleytronics ${ }^{4,5}$. Carrier-guiding quasi-one-dimensional (1D) graphene nanoribbons (GNRs) (-12 $^{6}$ with quantized energy subbands preserving the intrinsic Dirac nature have provided an ideal system to that end. Here, by guiding carriers through dual-gate operation in high-mobility monolayer graphene, we report the realization of quantized conductance in steps of $4 e^{2} / h$ in zero magnetic field, which arises from the full symmetry conservation of quasi-1D ballistic GNRs with effective zigzag-edge conduction. A tight-binding model calculation confirms conductance quantization corresponding to zigzag-edge conduction even for arbitrary GNR orientation. Valley-symmetry conservation is further confirmed by intrinsic conductance interference with a preserved Berry phase of $\pi$ in a graphene-based Aharonov-Bohm (AB) ring prepared by similar dual gating. This top-down approach for gate-defined carrier guiding in ballistic graphene is of particular relevance in the efforts towards efficient and promising valleytronic applications.

Valley-symmetry conservation in graphene nanoribbons (GNRs) has not yet been fully achieved. GNRs obtained by dry etching or grown naturally by chemical vapour deposition have exhibited transport characteristics with valley mixing or formation of an energy gap within the diffusive transport channels, which is most likely caused by random disorder ${ }^{13-16}$. Even suspended GNRs with reduced random disorder have exhibited conductance quantization $^{17}$ in steps of $2 e^{2} / h$ (where $e$ is the electron charge and $h$ is Planck's constant), which suggests broken valley symmetry in the ballistic one-dimensional (1D) transport channels of the system. Gate-defined guiding of non-relativistic carriers in bilayer graphene has also been attempted, but again with conductance quantization $^{18,19}$ in steps of $2 e^{2} / h$. More recently, $1 \mathrm{D}$ guiding of carriers has been introduced by electronic total reflection employing electrical means, which mimics fibre optics ${ }^{20,21}$. However, the scheme suffers from low efficiency of carrier guiding and does not show sufficient performance for adoption in valleytronic applications.

Valley-symmetry conservation requires effective suppression of intervalley scattering arising from short-range scattering from disorder such as atomically sharp defects, substrate roughness, and edge irregularities ${ }^{22,23}$. In this study, we have overcome these obstacles to obtaining rich intrinsic physics related to valley symmetry in transport measurements by taking several measures during device fabrication. We minimized scattering by atomically sharp defects and substrate roughness by sandwiching a monolayer graphene sheet between clean hexagonal boron nitride (hBN) layers ${ }^{24}$, and eliminated scattering by edge irregularities by gatedefined guiding of carriers instead of physically tailoring carriertransport paths in graphene. This unique carrier-guiding scheme leads to the practical realization of quasi-1D ballistic GNRs that conserve the intrinsic symmetries of graphene as in zigzag-edged GNRs. The scheme enables the exploration of graphene-based valleytronics and the investigation of phase-coherent transport characteristics governed by the intrinsic Dirac nature of graphene. These have not been explored thoroughly in previous studies based on diffusive GNRs with etch-defined physical paths ${ }^{25-27}$ of carrier transport.

In this study, we prepared both back- and top-gated ballistic graphene devices, wherein a monolayer graphene sheet was encapsulated by two thin crystalline hBN layers using a dry transfer method (see Methods and Fig. 1a). Through the dual operation of the back gate and top gates, the conductance map shown in Fig. 1b was obtained at $T=0.15 \mathrm{~K}$. The diagonal boundary between the unipolar and bipolar regions, corresponding to the local minimum conductance, tracks the charge neutral point (CNP) in the topgated region.

Electrostatic simulations based on finite-element analysis confirmed that the carrier type and the density $n$ in the globally back-gated and the locally top-gated graphene regions (regions $\mathrm{A}$ and B in Fig. 1c) can be independently fine-tuned by applying gate voltages $V_{\mathrm{bg}}$ and $V_{\mathrm{tg}}$, respectively. The density profiles shown in Fig. 1c correspond to the points denoted by the circular symbols in Fig. $1 \mathrm{~b}$ (colours match in the two figures). Although graphene has no intrinsic energy gap at the CNP, effective carrier guiding was attained along the quasi-1D transport channels between two adjacent top gates, with a sharp difference in the gated potential (or in corresponding $n$ ) as the Fermi level $E_{\mathrm{F}}$ of region $\mathrm{B}$ was tuned to the CNP whereas that of region A was off-tuned from the CNP. However, due to the potential gradient existing at the boundary of $\mathrm{A}$ and $\mathrm{B}$, the effective channel width $W^{*}$ experienced by carriers becomes narrower than the lithographically defined physical channel width $W$ (see Fig. 1c). The potential gradient over a scale larger than the lattice constant of graphene helps retain the valley symmetry for the carrier transport in a gate-defined GNR compared to the atomically sharp boundary of a physically etched GNR.

Figure 2a,b shows the conductance of the device as a function of Fermi wavevector $k_{\mathrm{F}}$, and the corresponding transconductance $\mathrm{d} G / \mathrm{d} k_{\mathrm{F}}$, which emphasizes features in the conductance $G$ itself. The most interesting characteristics of Fig. 2a,b are that the observed conductance is quantized in multiples of $\sim 4 e^{2} / h$, starting from

${ }^{1}$ Department of Physics, Pohang University of Science and Technology, Pohang 790-784, Republic of Korea. ${ }^{2}$ Advanced Materials Laboratory,

National Institute for Materials Science, 1-1 Namiki, Tsukuba 305-0044, Japan. *e-mail: hjlee@postech.ac.kr 
a
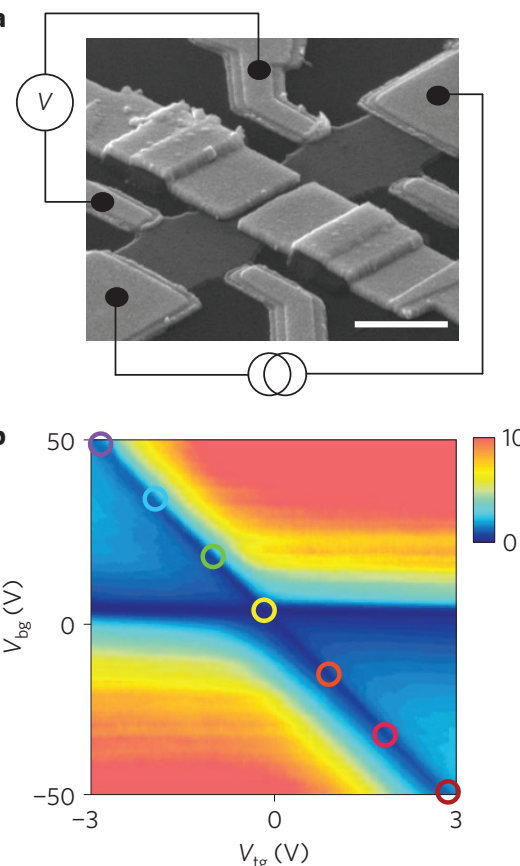

C

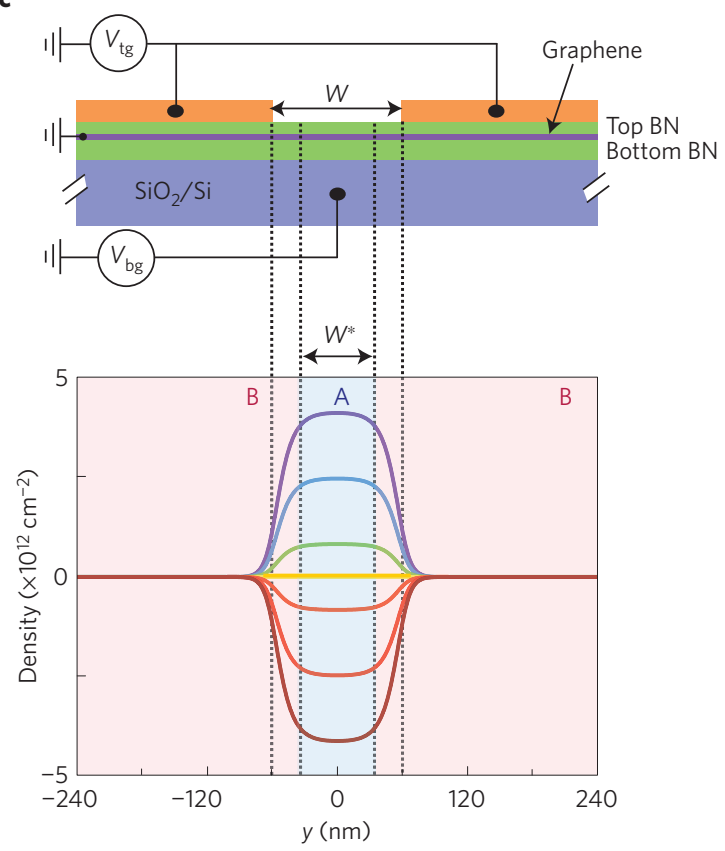

Figure 1 | Gate-defined quasi-1D transport channel built on graphene. a, Scanning electron microscopy (SEM) image of the device to verify the guiding of charge carriers with dual-gate operation. Top gates were deposited on top of a hexagonal boron nitride (hBN)/graphene/hBN device with standard Hall-bar geometry. Scale bar, $1 \mu \mathrm{m}$. b. Differential conductance $G$ as a function of back-gate voltage $V_{\mathrm{bg}}$ and top-gate voltage $V_{\text {tg }}$ measured at temperature $T=0.15 \mathrm{~K}$ in zero magnetic field. Coloured circular symbols on the diagonal minimum-conductance boundary between the monopolar and bipolar regions correspond to the same-coloured carrier density profile shown in c. c, Numerical calculation (lower panel) of carrier density for gate operation corresponding to the schematic device configuration (upper panel). By controlling both $V_{\mathrm{bg}}$ and $V_{\mathrm{tg}}$, the Fermi level of graphene under the top gate was tuned to stay on the charge neutral state. The distance between two top gates $W$ was $\sim 120 \mathrm{~nm}$, with $W^{*}(\sim 65 \mathrm{~nm})$ being the effective channel width estimated from the observed conductance profile.

$10 e^{2} / h$, which stems from the full conservation of both spin and valley symmetries in graphene. Previously, only conductance quantization in steps of $2 e^{2} / h$ or appreciably smaller values ${ }^{13-19,21}$ has been reported. The discrepancy in earlier studies could have been caused by edge disorder, low transmission probability in the conducting channels, or a possible change in the electronic structure in physically tailored GNR structures ${ }^{10-12}$. In our devices, the graphene sheet was free of structural deformation or corresponding changes in the electronic structure. Only the potential difference at the boundary between the top gate and the graphene guides the flow of carriers (more details will be discussed later, in relation to Fig. 3). The observed zero-field conductance quantization in multiples of $\sim 4 e^{2} / h$ in Fig. 2 demonstrates the first realization of quasi-1D ballistic conducting channels in graphene with full preservation of spin and valley symmetries.

We estimated the value of $W^{*}$ using the semi-classical relationship $G=4 e^{2} / \pi h \cdot k_{\mathrm{F}} W^{*}$ (dashed line in Fig. 2a). Values of $W^{*} \sim 65 \mathrm{~nm}$ for holes (Fig. 2a) and $52 \mathrm{~nm}$ for electrons (not shown) were obtained. Conductance quantization was reproduced in separate measurements on other devices with similar geometries (see Supplementary Information). The semi-classical expression is valid for $l_{\mathrm{e}} \gg W^{*}\left(l_{\mathrm{e}}\right.$; mean free path) and Fermi wavelengths ${ }^{28} \lambda_{\mathrm{F}}<2 W^{*}$. The former condition was easily satisfied and the latter condition was also met for $\left|V_{\mathrm{bg}}-V_{\mathrm{CNP}}\right|>2 \mathrm{~V}$ (or $k_{\mathrm{F}}>63 \times 10^{6} \mathrm{~m}^{-1} ; V_{\mathrm{CNP}}=1.4 \mathrm{~V}$ for the back gate). For a larger $\lambda_{\mathrm{F}}$ in the range $\left|V_{\mathrm{bg}}-V_{\mathrm{CNP}}\right|<2 \mathrm{~V}$, however, the quantum effect was disturbed and the conductance did not follow the semi-classical relationship. It is believed that this, together with weakened carrier guiding near the CNP, suppressed the expected conductance quantization for $G=2,6 e^{2} / h$ in our measurements.

The quantized channel conductance in steps of $4 e^{2} / h$ as 10,14 , $18,22, \ldots$ times $e^{2} / h$ (supposedly starting from $G=2 e^{2} / h$ ) observed here is a typical characteristic of ballistic zigzag-edged GNRs ${ }^{6-12}$. These robust zigzag-edge conduction characteristics in our GNRs are highly interesting, because no specific orientation was chosen for the quasi-1D channel between two adjacent top gates. It is highly unlikely that all the quasi-1D channels in our devices were aligned parallel to the zigzag-edge direction. Instead, it is highly probable that both zigzag- and armchair-like edges were mixed together randomly.

To confirm the characteristics of the robust zigzag-edged GNRs, we modelled the effective 1D transport channels confined by dualgate operation as GNRs. The electronic structure and quantum conductance of the GNRs were calculated using the tight-binding method. First-principles calculations were also performed to examine the validity of the tight-binding calculations (see Methods and Supplementary Fig. 7). Considering only one $\pi$ electron per carbon, the tight-binding Hamiltonian of GNRs is given by: $H=\varepsilon \sum_{i} c_{\mathrm{i}}^{\dagger} c_{\mathrm{i}}-t \sum_{\langle i j\rangle}\left(c_{\mathrm{i}}^{\dagger} c_{\mathrm{j}}+\right.$ h.c. $)$, where $\varepsilon$ is the on-site energy, $t$ is the nearest-neighbour hopping parameter, $c_{\mathrm{i}}^{\dagger}\left(c_{\mathrm{i}}\right)$ is the creation (annihilation) operator and h.c. is the Hermitian conjugate. Because the potential variation in the effective channel is not significant, the modulation in on-site and hopping terms was neglected in our calculations (for the effect of the boundary smoothness on the conductance, see Methods and Supplementary Fig. 9). The quantum conductance was calculated using the Landauer-Buttiker formalism: $G(E)=2 e^{2} / h T_{\mathrm{R}}(E)$, where the transmission coefficient $T_{\mathrm{R}}(E)$ is obtained using the recursive Green's function method ${ }^{29}$. In our calculations, the semi-infinite left and right leads and the central conductor are all GNRs. As shown in Fig. 1c, electronic transport is achieved in the $1 \mathrm{D}$ effective channel with width $W^{*}$.

The translational vector $\boldsymbol{T}_{m n}=m \boldsymbol{a}_{1}+n \boldsymbol{a}_{2} \equiv(m, n)$ with integers $m$ and $n$ (Fig. 3a) characterizes the axial orientation of GNRs. The zigzag-edged (armchair-edged) GNRs have $\boldsymbol{T}_{01}\left(\boldsymbol{T}_{11}\right)$. All other 
a

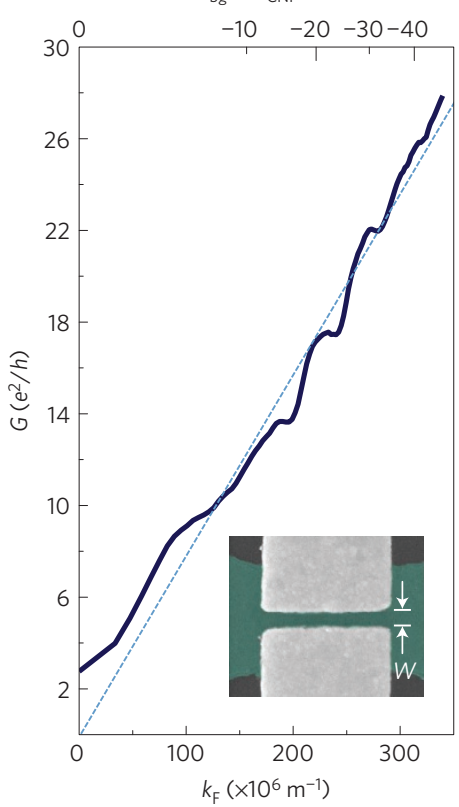

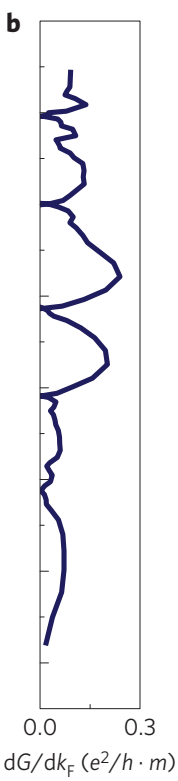

Figure 2 | Quantized conductance of a quasi-1D graphene channel in zero magnetic field. $\mathbf{a}, G$ as a function of the Fermi wavevector $k_{F}$ and $V_{b g}$ (or the carrier density $n ; V_{\mathrm{bg}}-V_{\mathrm{CNP}}=-10 \mathrm{~V}$ corresponds to $n=8.2 \times 10^{11} \mathrm{~cm}^{-2}$ ) for holes, with clear quantization observed at $10,14,18,22,26 \mathrm{e}^{2} / \mathrm{h}$ at $T=0.15 \mathrm{~K}$. The dashed line is a fit using the semi-classical relation $\mathrm{G}=4 \mathrm{e}^{2} / \mathrm{h} \cdot \mathrm{k}_{\mathrm{F}} W / \pi$ in the ballistic transport regime, which gives the effective channel width $W^{*} \sim 65 \mathrm{~nm}$. The inset shows the SEM image of the top gate deposited on the hBN/graphene/hBN stack (graphene is denoted in blue). The physical channel width between the two top gates, $W$, was $120 \mathrm{~nm}$. b, Variation of the transconductance $\mathrm{dG} / \mathrm{dk}_{\mathrm{F}}$. The minima, nearly periodic in $4 e^{2} / h$, correspond to the appearance of plateaux in the conductance trace. directions fall in between these two vectors. Atomic structures of GNRs together with $\boldsymbol{T}_{m n}$ are presented in the Supplementary Fig. 6 .

Figure $3 \mathrm{~b}$ shows calculated band structures of 60 -nm-wide GNRs for various axial orientations. The position of the valleys in the 1D Brillouin zone (BZ) is determined by the zone-folding scheme ${ }^{6}$ (Fig. 3c). The two inequivalent valleys $\left(\mathrm{K}\right.$ and $\left.\mathrm{K}^{\prime}\right)$ of graphene are projected onto $k= \pm l(m-n) / 3$ in the $1 \mathrm{D} \mathrm{BZ}$ with $l=2 \pi /\left|\boldsymbol{T}_{m n}\right|$, the size of the $1 \mathrm{D}$ BZ. If $m-n$ is not a multiple of three, $\mathrm{K}$ and $\mathrm{K}^{\prime}$ are projected onto different $\mathrm{k}$ points, which guarantees suppression of the intervalley scattering by crystal momentum conservation. Otherwise (that is, for $(1,4),(2,5)$ and $(1,1)$ orientations), the two valleys are projected onto the $\Gamma$ point (see Fig. $3 b$ ), which in principle can lead to intervalley scattering. However, even in this case of arbitrarily oriented GNRs, the boundary conditions ${ }^{7,9,11}$ suppress the intervalley scattering, except in the case of armchairedged boundary conditions. The imbalance in the number of sublattices at the edge sites (see Supplementary Fig. 6) decouples the states at the $\mathrm{K}$ and $\mathrm{K}^{\prime}$ valleys, leading to the zigzag-like boundary conditions imposing suppression of the valley mixing ${ }^{9}$. The only exception is all-armchair-edged GNRs, and thus the conductance step of $4 e^{2} / h$ occurs for arbitrarily oriented GNRs. The valley mixing in $1 \mathrm{D}$ conducting channels in graphene can be understood in terms of the crystal momentum and pseudospin conservations (see Supplementary Information and Supplementary Fig. 10).

Calculated band structures and quantum conductance for a $(1,2)$ oriented GNR are plotted in Fig. 3d and for a $(1,4)$-oriented GNR in Fig. 3e. We clearly observe a conductance step of $4 e^{2} / h$, two units of quantum conductance each from non-mixed valleys (for other types of GNR, see Supplementary Fig. 8). The conductance of $2 e^{2} / h$ near the CNP is the contribution from the edge-localized flat band. All these results demonstrate that arbitrarily oriented graphene quasi$1 \mathrm{D}$ channels defined with gate operation have the same conducting properties as zigzag-edged GNRs.

To further confirm the effectiveness of the conservation of valley symmetry in graphene along the specified guiding path, a

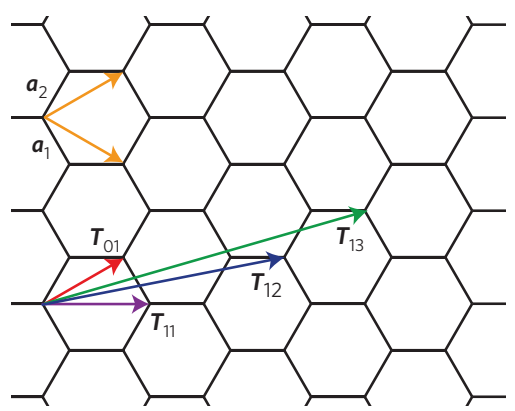

c

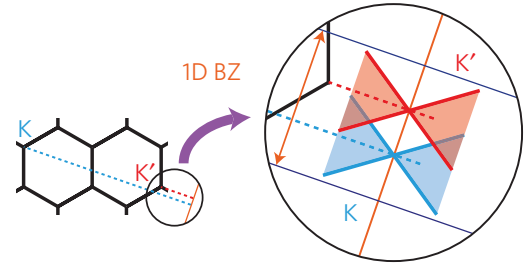

b
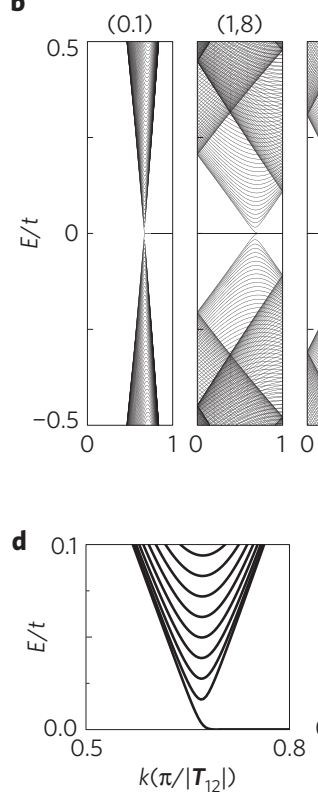
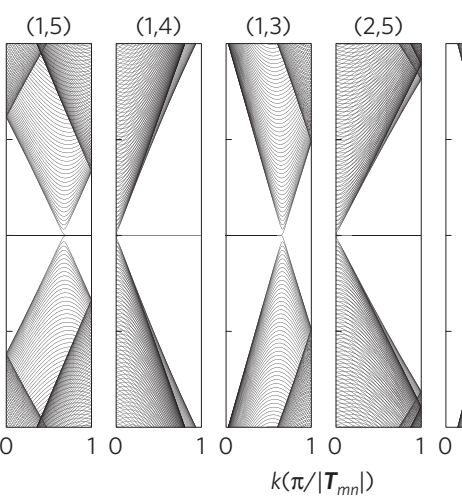

$(1,2)$
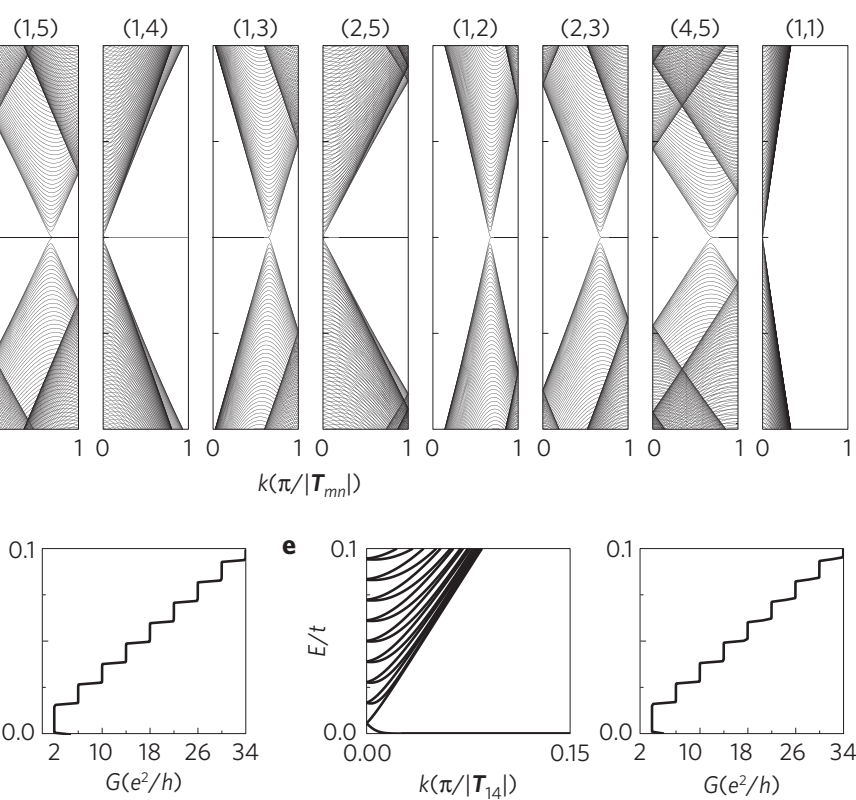

Figure 3 | Tight-binding calculations for GNRs with arbitrary axial direction. $\mathbf{a}$, Hexagonal lattice of graphene. $\boldsymbol{a}_{1}$ and $\boldsymbol{a}_{2}$ are the unit vectors. $\boldsymbol{T}_{\boldsymbol{m} \boldsymbol{n}}=\boldsymbol{m} \boldsymbol{a}_{1}+n \boldsymbol{a}_{2} \equiv(m, n)$ is the translational vector that characterizes the axial direction of GNRs. $\mathbf{b}$, Calculated band structures of 60 -nm-wide GNRs for various axial directions, as denoted at the top of the panels. Leftmost panel, $(0,1)$ zigzag-edged GNR and rightmost panel, $(1,1)$ armchair-edged GNR. c, Zone folding of the graphene 2D BZ into a 1D BZ of $(1,2)$ GNR. Projections of the Dirac valleys at K (blue cone) and $K^{\prime}$ (red cone) into 1D BZ (solid line in orange) are illustrated in the enlarged circle. d,e, The band structure (left panel) and quantum conductance (right panel) of 60-nm-wide GNR with (1,2) (d) and $(1,4)(\mathbf{e})$ axial direction. The quantum conductance reveals a step of $4 e^{2} / h$, the same as for the zigzag-edged GNR. 


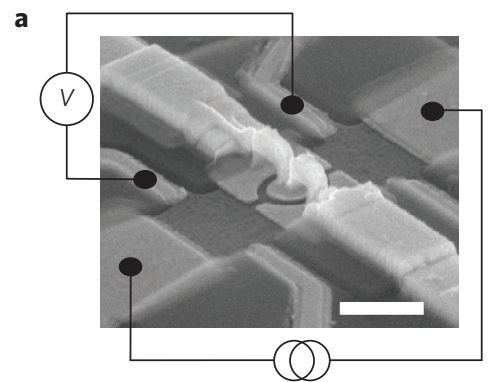

b

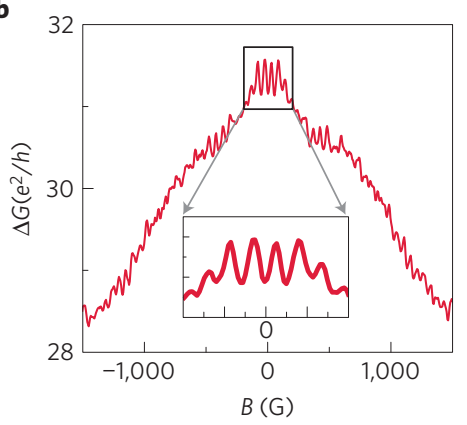

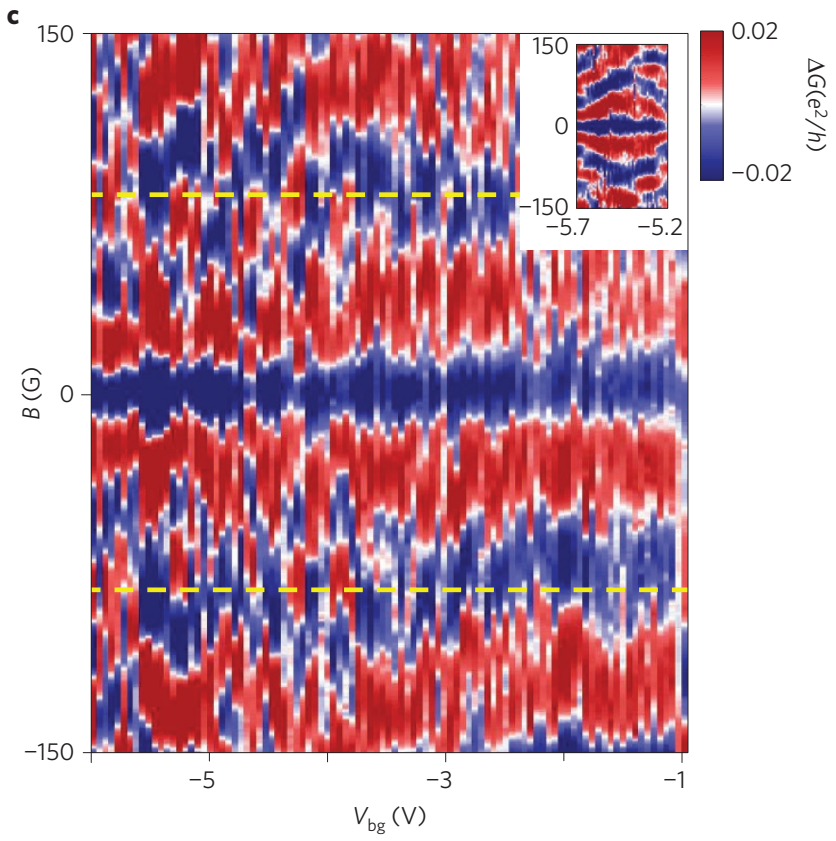

Figure 4 | Aharonov-Bohm interference in a ballistic graphene device. a, SEM image of the device showing the Aharonov-Bohm (AB) interference. Ring-shaped top gates are connected together by air bridging. Scale bar, $1 \mu \mathrm{m}$. $\mathbf{b}$, Negative magnetoconductance of the interferometer at $T=0.15 \mathrm{~K}$ for $V_{\mathrm{bg}}=30 \mathrm{~V}$. Periodic oscillations are clearly visible in the inset. $\mathbf{c}, \Delta G$ as a function of $V_{\mathrm{bg}}$ and $B$. $A$ persistent conductance minimum exists at $B=0$, pointing to the robust destructive interference over a wide range of $V_{b g}$. Bands of blueish regions around the dotted lines correspond to the first oscillation minima. The inset, taken from a separate high-resolution scan in a narrower $V_{\text {bg }}$ range for the same device, reveals continuously varying AB oscillations.

Aharonov-Bohm (AB) interferometry was investigated with an $\mathrm{AB}$ ring defined by dual-gate operation similar to that introduced above. Figure $4 \mathrm{a}$ presents a typical $\mathrm{AB}$ interferometer in a four-terminal configuration. The central circular top gate and the two nearby side top gates were connected together by air bridging, which in turn were connected to outside gating leads.

Figure $4 \mathrm{~b}$ shows the four-terminal magnetoconductance across the $\mathrm{AB}$ ring at $V_{\mathrm{bg}}=-30 \mathrm{~V}$, with the background exhibiting a negative magnetoconductance. This arose from the enhanced backscattering rate of carriers with increasing magnetic field, which led to weak anti-localization ${ }^{30}$. This result suggests that the quasi$1 \mathrm{D}$ transport channels formed in our device preserved the valley symmetry with negligible intervalley scattering. Clear periodic oscillations can be seen on top of the background (Fig. 4b), with a magnetic-field period $\Delta B=56 \mathrm{G}$. The period corresponds to one flux quantum $h / e$ threading the $\mathrm{AB}$ ring of $485 \mathrm{~nm}$ in radius, which is in good agreement with the mean radius $r=470 \mathrm{~nm}$ of the ring, estimated on the basis of scanning electron microscopy (see the detailed fast Fourier-transform analysis in Methods and Supplementary Fig. 12).

Details of the AB interference (see inset Fig. 4b) reveal destructive interference for zero field, which arises from valley-symmetrypreserving transport together with acquisition of a Berry phase of $\pi$. This Berry phase is revealed in the wavefunction of a carrier when it encloses the degeneracy point in momentum space without intervalley scattering. In Fig. $4 \mathrm{c}$, the destructive $\mathrm{AB}$ oscillations for zero field persist near the CNP in a wide $V_{\mathrm{bg}}$ range, between -6 and $-1 \mathrm{~V}\left(V_{\mathrm{CNP}}=-0.5 \mathrm{~V}\right)$. This finding clearly demonstrates that the zero-field quantum phenomenon with a Berry phase of $\pi$ is closely related to the relativistic nature of carriers in graphene. A large variation of the Fermi wavevector within this range of $V_{\mathrm{bg}}$ caused a significant change in the propagation routes of carrier partial waves in the $A B$ ring, which led to an irregular but generally continuous variation of the oscillation, except for $B=0$. However, robust preservation of the valley symmetry in our gate-defined graphene-based $\mathrm{AB}$ interferometer leads to persistent destructive interference at zero field.

\section{Methods}

Methods, including statements of data availability and any associated accession codes and references, are available in the online version of this paper.

Received 19 December 2015; accepted 25 May 2016; published online 20 June 2016

\section{References}

1. Castro Neto, A. H., Guinea, F., Peres, N. M. R., Novoselov, K. S. \& Geim, A. K. The electronic properties of graphene. Rev. Mod. Phys. 81, 109-162 (2009).

2. McCann, E. et al. Weak-localization magnetoresistance and valley symmetry in graphene. Phys. Rev. Lett. 97, 146805 (2006).

3. Trushin, M. \& Schliemann, J. Pseudospin in optical and transport properties of graphene. Phys. Rev. Lett. 107, 156801 (2011).

4. Rycerz, A., Tworzydlo, J. \& Beenakker, C. W. J. Valley filter and valley valve in graphene. Nature Phys. 3, 172-175 (2007).

5. Qiao, Z., Yang, S. A., Wang, B., Yao, Y. \& Niu, Q. Spin-polarized and valley helical edge modes in graphene nanoribbons. Phys. Rev. B 84, 035431 (2011).

6. Nakada, K., Fujita, M., Dresselhaus, G. \& Dresselhaus, M. S. Edge state in graphene ribbons: nanometer size effect and edge shape dependence. Phys. Rev. B 54, 17954-17961 (1996).

7. Brey, L. \& Fertig, H. A. Electronic states of graphene nanoribbons studied with the Dirac equation. Phys. Rev. B 73, 235411 (2006).

8. Peres, N. M. R., Castro Neto, A. H. \& Guinea, F. Conductance quantization in mesoscopic graphene. Phys. Rev. B 73, 195411 (2006).

9. Akhmerov, A. R. \& Beenakker, C. W. J. Boundary conditions for Dirac fermions on a terminated honeycomb lattice. Phys. Rev. B 77, 085423 (2008).

10. Li, T. C. \& Lu, S.-P. Quantum conductance of graphene nanoribbons with edge defects. Phys. Rev. B 77, 085408 (2008).

11. Wurm, J., Wimmer, M. \& Richter, K. Symmetries and the conductance of graphene nanoribbons with long-range disorder. Phys. Rev. B 85, 245418 (2012).

12. Orlof, A., Ruseckas, J. \& Zozoulenko, I. V. Effect of zigzag and armchair edges on the electronic transport in single-layer and bilayer graphene nanoribbons with defects. Phys. Rev. B 88, 125409 (2013). 
13. Lin, Y.-M., Perebeinos, V., Chen, Z. \& Avouris, P. Electrical observation of subband formation in graphene nanoribbons. Phys. Rev. B 78, 161409 (2008).

14. Stampfer, C. et al. Energy gaps in etched graphene nanoribbons. Phys. Rev. Lett. 102, 056403 (2009).

15. Lian, C. et al. Quantum transport in graphene nanoribbons patterned by metal masks. Appl. Phys. Lett. 96, 103109 (2010).

16. Baringhaus, J. et al. Exceptional ballistic transport in epitaxial graphene nanoribbons. Nature 506, 349-354 (2014)

17. Tombros, N. et al. Quantized conductance of a suspended graphene nanoconstriction. Nature Phys. 7, 697-700 (2011).

18. Goossens, A. M. et al. Gate-defined confinement in bilayer graphenehexagonal boron nitride hybrid devices. Nano Lett. 12, 4656-4660 (2012).

19. Allen, M. T., Martin, J. \& Yacoby, A. Gate-defined quantum confinement in suspended bilayer graphene. Nature Commun. 3, 934 (2012).

20. Williams, J. R., Low, T., Lundstrom, M. S. \& Marcus, C. M. Gate-controlled guiding of electrons in graphene. Nature Nanotech. 6, 222-225 (2011).

21. Rickhaus, P. et al. Guiding of electrons in a few-mode ballistic graphene channel. Nano Lett. 15, 5819-5825 (2015).

22. Ki, D.-K., Jeong, D., Choi, J.-H., Lee, H.-J. \& Park, K.-S. Inelastic scattering in a monolayer graphene sheet: a weak-localization study. Phys. Rev. B 78, 125409 (2008).

23. Tikhonenko, F. V., Kozikov, A. A., Savchenko, A. K. \& Gorbachev, R. V. Transition between electron localization and antilocalization in graphene. Phys Rev. Lett. 103, 226801 (2009).

24. Wang, L. et al. One-dimensional electrical contact to a two-dimensional material. Science 342, 614-617 (2013).

25. Russo, S. et al. Observation of Aharonov-Bohm conductance oscillations in a graphene ring. Phys. Rev. B 77, 085413 (2008)

26. Magdalena, H. et al. The Aharonov-Bohm effect in a side-gated graphene ring. New J. Phys. 12, 043054 (2010).
27. Kim, M. et al. Tuning locality of pair coherence in graphene-based Andreev interferometers. Sci. Rep. 5, 8715 (2015).

28. van Wees, B. J. et al. Quantized conductance of point contacts in a two-dimensional electron gas. Phys. Rev. Lett. 60, 848-850 (1988).

29. Li, T. et al. Effects of dangling ends on the conductance of side-contacted carbon nanotubes. Phys. Rev. B 72, 035422 (2005).

30. Beenakker, C. W. J. \& van Houten, H. Boundary scattering and weak localization of electrons in a magnetic field. Phys. Rev. B 38, 3232-3240 (1988).

\section{Acknowledgements}

We would like to thank G.-H. Lee, G.-H. Park, J.-H. Lee and J. Lee for useful discussions on the device fabrication. This work was supported by the National Research Foundation (NRF) through the SRC Center for Topological Matter (Grant No. 2011-0030046 for H.J.L. and Grant No. 2011-0030789 for S.H.J.) and the GFR Center for Advanced Soft Electronics (Grant No. 2014M3A6A5060956).

\section{Author contributions}

M.K. and H.-J.L. conceived the idea and designed the experiments. M.K. prepared the samples and performed measurements. M.K. and H.-J.L. analysed the data. J.-H.C., S.-H.L. and S.-H.J. provided the theoretical consultation and calculation. K.W. and T.T. supplied the high-quality hexagonal boron nitride. H.-J.L. supervised the study. M.K., J.-H.C., S.-H.L., S.-H.J. and H.-J.L. wrote the manuscript. All authors contributed to the discussion and reviewed the manuscript.

\section{Additional information}

Supplementary information is available in the online version of the paper. Reprints and permissions information is available online at www.nature.com/reprints. Correspondence and requests for materials should be addressed to H.-J.L.

\section{Competing financial interests}

The authors declare no competing financial interests. 


\section{Methods}

Sample fabrication. Monolayer graphene was encapsulated between two relatively thin (typically thinner than $30 \mathrm{~nm}$ ) crystals of hBN using the dry transfer technique $^{24}$. This structure was placed onto a heavily electron-doped Si substrate capped with a 300-nm-thick oxidation layer. The standard electron-beam lithography process was then adopted to apply contact electrodes to the heterostructure with the following sequence: electron (e)-beam patterning of contact regions, plasma etching to make trenches, e-gun deposition of electrodes and the lift-off process. Electrodes were prepared by in situ sequential deposition of $\mathrm{a} \mathrm{Cr} / \mathrm{Au}(10 \mathrm{~nm} / 50 \mathrm{~nm})$ bilayer. The top gates were then deposited directly onto the top $\mathrm{hBN}$ to make a sharp potential gradient. The sides of the heterostructure were electrically insulated with an $\mathrm{Al}_{2} \mathrm{O}_{3}$ layer before depositing the top gates, because the top-gate electrode could provide unintended electrical shorting to the side of the heterostructure. This insulation was located far from the actual transport channels, to ensure that it did not affect the transport characteristics related to top gating.

For the $\mathrm{AB}$ interferometer, the side top gates were connected to the central top-gate island by air bridging combined with a multilevel lithography process ${ }^{31}$. A LOR 3A (MicroChem) resist layer was spin-coated at 4,000 r.p.m. for 30 s, and then baked on a hot plate at $200^{\circ} \mathrm{C}$ for $90 \mathrm{~s}$. This layer was used as a temporary support for the suspended portion of the air bridge. Following the processes of making connections between top gates, involving standard e-beam lithography, e-gun evaporation, and lift-off, the LOR layer was carefully removed, leaving the air-bridge-connected top gates. The suspension height of the air bridge above the top hBN surface was measured to be $\sim 350 \mathrm{~nm}$. Considering the small dielectric constant of vacuum, the air bridge had a negligible effect on the carrier modulation in graphene underneath during top-gate operation.

Device geometry. For the GNR device discussed in the main text, the total length between the source and drain electrodes was $\sim 3.8 \mu \mathrm{m}$. The centre-to-centre distance between the lateral voltage probes was $\sim 2.2 \mu \mathrm{m}$. Lithographically defined channel width and length of the GNR between top gates were $\sim 120 \mathrm{~nm}$ and $\sim 1.1 \mu \mathrm{m}$, respectively. In the AB-ring interferometer device, the total length between the source and drain electrodes was $4.4 \mu \mathrm{m}$. The centre-to-centre distance between lateral voltage probes was $\sim 2.7 \mu \mathrm{m}$. The channel width and the average radius of the $\mathrm{AB}$ ring were $\sim 150 \mathrm{~nm}$ and $470 \mathrm{~nm}$, respectively. See the SEM image of Supplementary Fig. 1.

Measurements. Application of a voltage $V_{\mathrm{bg}}$ to the $\mathrm{Si}$ substrate, underneath the 280-nm-thick $\mathrm{SiO}_{2}$ and a 20-30-nm-thick bottom hBN connected in series, allowed tuning of the overall carrier density $n$ in the graphene devices. With the top gate deposited directly on the 10-30-nm-thick top hBN layer, $n$ was locally modulated in the graphene under the top gate by application of a voltage $V_{\mathrm{tg}}$. Electrical characterization of the devices was performed using a standard lock-in technique operated at a frequency of $13.3 \mathrm{~Hz}$ with an a.c. bias current of $10 \mathrm{nA}$. The graphene layer employed in this study showed carrier mobilities ranging from 50,000 to $300,000 \mathrm{~cm}^{2} \mathrm{~V}^{-1} \mathrm{~s}^{-1}$, extracted from the conductivity at $n \sim 10^{12} \mathrm{~cm}^{-2}$ at $T=0.15 \mathrm{~K}$. The residual carrier density from the electron-like and hole-like puddles was approximately $10^{10} \mathrm{~cm}^{-2}$. Also, from the negative resistance observed in a van der Pauw configuration, we confirmed that graphene used in our study was in a true ballistic transport regime with sufficiently long $l_{\mathrm{e}}$ over the scales of the device $\sim 1.1 \mu \mathrm{m}$. As shown in Fig. 1b, the slope of the diagonal boundary between the monopolar and bipolar regions $(\sim-15.7)$ leads to an estimation of the thickness of the top hBN layer of $\sim 19 \mathrm{~nm}$, where the slope is equal to the ratio of the dielectric capacitances of the top gate and back gate. The thin top hBN layer enabled an interface of sharp potential difference to be set up.

Details of tight-binding calculations. We performed first-principles calculations based on the density functional theory implemented in the Quantum Espresso package $^{32}$ to check the validity of the tight-binding method. We used the ultrasoft pseudopotential to describe the electron-ion interaction. The exchange-correlation energy was treated within the generalized gradient approximation (GGA) parameterized by Perdew, Burke and Ernzerhof ${ }^{33}$. The energy cutoff for the expansion of the plane-wave basis set was 32 Ry. The Brillouin zone integration was done with the $k$-point sampling at $1 \times 30 \times 1$ grid for zigzag-edged GNRs and at
$1 \times 15 \times 1$ grid for armchair-edged GNRs. The edge atoms of GNRs were passivated by hydrogen atoms. The Hamiltonian for the band structures was constructed from the basis of the maximally localized Wannier function ${ }^{34}$ (MLWFs) implemented in the Wannier90 (ref. 35). The Green's function to calculate the quantum conductance was also obtained from the Hamiltonian. The semi-infinite left and right leads and the central conducting region are all GNRs ${ }^{29,36}$.

Supplementary Fig. 7 shows the calculated band structures and quantum conductance obtained by both the first-principles and tight-binding methods. In our tight-binding calculations, only the nearest-neighbour hopping integral was considered and set to $t=2.7 \mathrm{eV}$ as in ref. 37. Despite the absence of higher-order hopping and edge-hopping modulations, the quantum conductance step of $2 e^{2} / h$ in armchair-edged GNRs (in panels a, b, e, f of Supplementary Fig. 7) and $4 e^{2} / h$ in zigzag-edged GNRs (in panels c, d, g, h) are well described.

Effect of smooth channel boundaries. To investigate the effect of the boundary smoothness on the conductance, we emulated the carrier density profile in Fig. 1c by incorporating potential profiles (Supplementary Fig. 9a) into our tight-binding Hamiltonian as the on-site energy term $\left(V\left(y_{\mathrm{i}}\right)\right.$ with $y_{\mathrm{i}}$ along the transverse direction in the channels), $H=\sum_{i} V\left(y_{\mathrm{i}}\right) c_{\mathrm{i}}^{\dagger} c_{\mathrm{i}}-\sum_{\langle i j\rangle}\left(c_{\mathrm{i}}^{\dagger} c_{\mathrm{j}}+\right.$ h.c. $)$. Smoothness of a boundary is modelled by a sine function $V\left(y_{\mathrm{i}}\right)$ near the GNR edge. The quantum conductance of a $15 \mathrm{~nm}$-width GNR along the zigzag and $(1,4)$ directions (without potential modification) is depicted in Supplementary Fig. $9 b, c$. The conductance step is $4 e^{2} / h$. From the semi-classical relation $G=\left(4 e^{2} / \pi h\right) k_{\mathrm{F}} W^{*}$ in the ballistic transport regime, the effective channel width is estimated to be $\sim 14 \mathrm{~nm}$. Supplementary Fig. 9d,e shows the conductance step of $4 e^{2} / h$ for the $1 D$ channels with the smooth boundaries, the same value of step as that for GNRs in Supplementary Fig. 9b,c. This indicates that the boundary smoothness does not disturb the conductance step, regardless of the degree of smoothness and the channel direction. The effective channel width is estimated to be $\sim 15 \mathrm{~nm}$. In this calculation, to reduce the computation time, we assume the size of GNR to be $W^{*} \sim 15 \mathrm{~nm}$, which is smaller than the actual value of $W^{*} \sim 65 \mathrm{~nm}$. The gate-defined GNR exhibit almost identical conductance plateaux as GNRs of the same channel width with sharp boundaries.

Fast Fourier-transform analysis on AB interference. In Supplementary Fig. 12, the Fourier spectrum of the magnetoconductance shown in Fig. $3 \mathrm{~b}$ is plotted. The peak seen at $1 / 56 \mathrm{G}^{-1}$ corresponds to the $h / e$-periodic $\mathrm{AB}$ effect. In the spectrum, the position and width of the $h / e$ peak correspond well to the expected $h / e$ oscillations, given the values of the inner and outer radii in our device (indicated as a pink shaded region in Supplementary Fig. 12). Higher harmonics, especially $h / 2 e$-periodic oscillations, are also visible in the Fourier spectrum. There exists a secondary peak of $\sim 9 \%$ of the primary $h / e$-periodic oscillation amplitude, verifying the long phase coherence in this system, as expected.

Data availability. The data that support the plots within this paper and other findings of this study are available from the corresponding author upon reasonable request.

\section{References}

31. Liu, G., Velasco, J., Bao, W. \& Lau, C. N. Fabrication of graphene p-n-p junctions with contactless top gates. Appl. Phys. Lett. 92, 203103 (2008).

32. Giannozzi, P. et al. QUANTUM ESPRESSO: a modular and open-source software project for quantum simulations of materials. J. Phys. Condens. Matter 21, 395502 (2009).

33. Perdew, J. P., Burke, K. \& Ernzerhof, M. Generalized gradient approximation made simple. Phys. Rev. Lett. 77, 3865-3868 (1996).

34. Marzari, N. \& Vanderbilt, D. Maximally localized generalized Wannier functions for composite energy bands. Phys. Rev. B 56, 12847-12865 (1997).

35. Charlier, J.-C., Blase, X. \& Roche, S. Electronic and transport properties of nanotubes. Rev. Mod. Phys. 79, 677-732 (2007).

36. Sancho, M. L., Sancho, J. L. \& Rubio, J. Highly convergent schemes for the calculation of bulk and surface Green functions. J. Phys. F 15, 851-858 (1985).

37. Hancock, Y., Uppstu, A., Saloriutta, K., Harju, A. \& Puska, M. Generalized tight-binding transport model for graphene nanoribbon-based systems. Phys. Rev. B 81, 245402 (2010). 